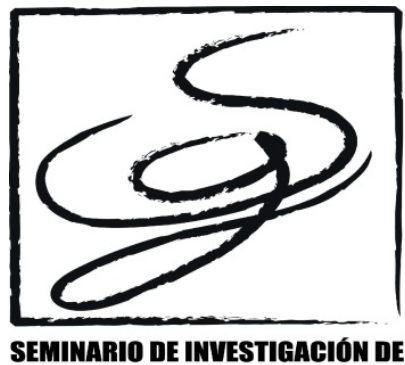

\title{
Pedagogía menstrual como herramienta para el empoderamiento femenino
}

\author{
Marina López García \\ Universidad Pablo de Olavide \\ marina.loga@hotmail.com \\ Xiana Pena Lima \\ Universidad Pablo de Olavide \\ xianapena@gmail.com
}

Fecha de recepción: 31/07/2020 Fecha de evaluación: 10/09/2020

Fecha de aceptación: 09/11/2020

\begin{abstract}
:
In our society, the application of feminist and gender perspectives to research and social conception of the menstrual cycle continues to be a pending task. In the collective insight, menstruation is still fraught with stereotypes and myths, due to the absence of educational, social, health and political resources for its treatment. This leads, on the one hand, into the perpetuation of the taboo that encompasses the menstrual experience, and on the other hand, in a lack of knowledge about its main characteristics, phases, symptoms and signs, associated pathologies, etc. This project aims to highlight the importance of self-knowledge, self-care and the ability to understand the signals from our bodies as personal resources of individual and collective empowerment, through awareness and creation of common learning and experiences in relation to the menstrual cycle.
\end{abstract}

Key-words: menstrual pedagogy; sex education; empowerment; gender; mainstreaming; feminism

\section{Resumen:}

La aplicación de perspectivas feministas y de género a la investigación y concepción social del ciclo menstrual continúa siendo una tarea pendiente en nuestra sociedad. En el imaginario social colectivo, la menstruación se presenta -todavía- como un elemento mitificado y estereotipado, debido a la carencia de recursos educativos, sociales, sanitarios y políticos destinados al tratamiento del mismo. Esto se traduce en la 
perpetuación del tabú que engloba a la experiencia de la menstruación y en el desconocimiento generalizado de la población acerca de sus principales características, fases, síntomas y signos, patologías asociadas, etc.

Con la presente propuesta de intervención se pretende resaltar la importancia del autoconocimiento, el autocuidado y la capacidad para entender las señales de nuestros cuerpos como recursos personales de empoderamiento individual y colectivo, a través de la toma de conciencia y creación de aprendizajes y experiencias comunes en relación con el ciclo menstrual.

Palabras clave: pedagogía menstrual; educación sexual; empoderamiento; género; mainstreaming; feminismo

\section{Introducción}

Hace ya tiempo que diversos/as autores/as y profesionales de distintos ámbitos ponen de manifiesto la necesidad de desarrollar la educación sexual integral de toda la población, pero, más aún, en la adolescencia y juventud. Barragán (1999) define la educación sexual como la herramienta para transmitir y reproducir los valores, normas y concepciones morales acerca de la sexualidad en cada cultura, por lo que depende del modelo sociopolítico y económico concreto.

En nuestra sociedad, a pesar de encontrarnos en la denominada «era de la información y comunicación», continúan dándose numerosos casos de personas que no se sienten a gusto con sus relaciones sexuales y que no saben cómo explorar y expresar su sexualidad. Se trata de un momento que, a nivel teórico, parece privilegiado para la adolescencia en cuanto a conocimientos anatómicos, fisiológicos y relacionados con los métodos anticonceptivos, que les permita gozar plenamente de una sexualidad más libre, más igualitaria para ambos sexos y sin embarazos no deseados o problemas de Infecciones de Transmisión Sexual (ITS). Sin embargo, en la práctica, nos seguimos encontrando con una realidad que se aleja de la deseable (López, 2004).

Al mismo tiempo, si se realiza un análisis de este problema desde una perspectiva de género, se observa que, en la juventud y adolescencia, se siguen reproduciendo numerosas actitudes machistas y patriarcales en las relaciones, tanto a nivel interpersonal y afectivo como a nivel erótico y sexual (Jorge, 2016). Los roles de género patriarcales se reproducen en absolutamente todos los ámbitos de la sociedad de la que formamos parte (Varela, 2013). Por este motivo, prevalece una concepción de las relaciones eróticas androcentrista y falocéntrica, en la que el placer del hombre, en muchas ocasiones, acaba siendo el fin último del encuentro sexual. Esto, además, se propicia desde los medios de comunicación, las películas, la industria pornográfica, etc., que, en gran parte de los casos, son los únicos referentes de cultura sexual con los que cuentan las personas en edad escolar (y toda la población). La 
realidad es que, tal y como afirman Soubies et al. (2010), lo relacionado con la sexualidad sigue siendo un tabú a día de hoy en muchas instituciones, familias y conversaciones formales e informales y todo lo que rodea al fenómeno de la menstruación no se encuentra exento.

\section{Método}

Para la realización del análisis de la realidad que precede al diseño de la intervención se plantea un diagnóstico desde un enfoque cualitativodescriptivo, a través de la aplicación de técnicas e instrumentos mixtos, con el diseño de un modelo de cuestionario y la realización de un grupo de discusión.

\subsection{Cuestionario dirigido a personas menstruantes}

Se diseña un cuestionario compuesto por 13 preguntas, en modalidades de respuesta tanto cerrada como abierta y relacionadas con los siguientes bloques temáticos:

1. Conocimiento general del ciclo menstrual.

2. Mitos y estereotipos interiorizados por las propias personas menstruantes.

3. Síntomas y métodos paliativos utilizados. Se distribuye mediante soporte electrónico (modalidad online) y va dirigido a una muestra de 100 personas menstruantes, con edades comprendidas entre los 16 y 25 años.

\subsection{Grupo de discusión sobre el ciclo menstrual}

Dirigido a las personas menstruantes y no menstruantes, para analizar la realidad en relación con el tema propuesto en la sociedad general. Se plantean las siguientes preguntas:

- ¿Cómo afecta la menstruación a las personas menstruantes?

- ¿Sigue siendo un tabú? ¿Cuáles son las creencias, ideas y opiniones que se tienen socialmente sobre ella?

- Educación sexual femenina: ¿existe?, ¿es suficiente?, ¿qué formación/información se echa en falta a día de hoy?

\section{Desarrollo}

El proyecto de intervención Somos una, somos cuatro pretende desarrollarse a través de una metodología abierta y flexible, basada en las necesidades, motivaciones e intereses de las personas destinatarias, siguiendo el hilo temático conductor propuesto en los bloques generales de contenido.

La intervención se llevará a cabo con un total de 24 personas menstruantes de entre 16 y 25 años, divididas en dos grupos de 12 personas cada uno. Se plantea esta división del total de personas por las características de las propias actividades diseñadas, basadas en la participación activa y la creación de conocimientos y experiencias 
colectivas en relación con el ciclo menstrual, mediante el uso de técnicas como debates, grupos de discusión, etc.

Se plantean un total de 23 actividades que se dividen en 21 sesiones-, incluyendo talleres de formación y de creación de material propio de información en materia del tema propuesto. La duración de las sesiones variará en función de la actividad, teniendo como mínimo duración de una hora y, como máximo, de hora y media.

Por otro lado, se pretende que las participantes tengan un papel activo en el proceso de aprendizaje, junto con las profesionales, evitando la mera transmisión de información teórica y fomentando un ambiente de conocimientos y experiencias compartidas, con el objetivo de favorecer el autoconocimiento y empoderamiento colectivo de las destinatarias.

Las actividades propuestas se desarrollarán siguiendo el orden de los siguientes bloques temáticos:

- Bloque temático 1: Desestigmatización, desmitificación y normalización de la menstruación y las características de sus diferentes fases.

- Bloque temático 2: Menstruación cultural: el ciclo menstrual en otras culturas.

- Bloque temático 3: Concienciación y divulgación de información relativa a la menstruación y las fases del ciclo menstrual.

- Bloque temático 4: Menstruación y salud pública.

- Bloque temático 5: La menstruación y los autocuidados.

Se plantean, además, actividades transversales que se llevarán a cabo desde el inicio hasta el final del desarrollo del proyecto, configurado como un proceso de aprendizaje gradual y acumulativo. Por ello, las participantes tendrán acceso a la información y a la bibliografía utilizada en todas las sesiones.

Asimismo, cobrará especial importancia la realización de una actividad de presentación al principio del proyecto y otra de cierre al término de este. Las dinámicas propuestas facilitarán la creación de un espacio propicio para el desarrollo de vínculos de confianza y apoyo entre las personas participantes, así como el intercambio de vivencias personales.

Para la puesta en práctica de la intervención se hará uso de diferentes técnicas, entre las cuales destacan: los grupos de discusión y el debate, el brainstorming y el uso de pequeños grupos de trabajo. El conjunto de la intervención tendrá una duración total de 9 meses.

\section{Resultados}

Las personas con vulva menstruantes constituyen, aproximadamente, la mitad de la población mundial, por lo que se consideran necesarios la creación y el fomento de recursos de formación e información - prácticamente inexistentes hasta el momento- en relación con el ciclo menstrual: sus características, síntomas y signos principales, opciones de tratamiento, estrategias para la identificación de los cambios producidos durante sus fases, etc. 
Los datos obtenidos revelan que la realidad social que engloba al proceso de la menstruación como parte de la salud sexual femenina continúa cargada de estereotipos, mitos y creencias falsas que se traducen en situaciones que infieren de manera significativa disminuyendo la calidad de vida de las personas menstruantes. Así, la reproducción y perpetuación de los tabúes asociados al ciclo menstrual generan situaciones de violencia estructural englobadas dentro del sistema patriarcal hacia el colectivo de personas menstruantes, en tanto que inciden en la concepción y tratamiento del propio ciclo y en las patologías asociadas al mismo.

En relación con las necesidades detectadas a través del análisis de la realidad, cabe destacar la escasa información sobre el ciclo menstrual, manteniendo ideas distorsionadas e influidas por la socialización de género y patriarcal que caracteriza nuestro contexto. Además, se observa una sobremedicalización de las personas participantes como estrategia paliativa de los síntomas, debido a la falta de información relacionada con las alternativas al tratamiento farmacológico y la salud sexual femenina en general, como se ha mencionado anteriormente.

El nivel de influencia de los síntomas provocados por la menstruación, por otro lado, es un indicativo de necesidad de intervención en este ámbito, debido a las consecuencias negativas que estos tienen en gran parte de las personas menstruantes (haciendo referencia, incluso, a presentar depresión o ideas de suicidio).

Por este motivo, la pedagogía menstrual se conforma como un movimiento innovador y necesario para la mitigación de las desigualdades y violencias sufridas por las personas menstruantes en nuestra sociedad, además de contribuir a la creación de identidades y experiencias compartidas que mejoren la calidad de vida de las personas menstruantes y sus relaciones sociales, contribuyendo a la equidad de género y al avance hacia sociedades más justas e igualitarias. 
Ambigua, Revista de Investigaciones sobre Género y Estudios

Culturales, n. ${ }^{\circ}$ 7, 2020, pp. 387-392. ISSN: 2386-8708 